\title{
KLIKK proteases of Tannerella forsythia: putative virulence factors with a unique domain structure
}

\author{
Miroslaw Ksiazek ${ }^{1 * t}$, Danuta Mizgalska ${ }^{1 * t}$, Sigrum Eick ${ }^{2}$, Ida B. Thøgersen ${ }^{3}$, \\ Jan J. Enghild ${ }^{3}$ and Jan Potempa ${ }^{1,4 *}$
}

OPEN ACCESS

Edited by:

Christina Maria Joseph Elisabeth Vandenbroucke-Grauls,

VU University Medical Center,

Netherlands

Reviewed by:

Magdalena Chirila,

Iuliu Hațieganu University of Medicine and Pharmacy, Romania

LiXu,

Cornell University, USA

${ }^{*}$ Correspondence:

Miroslaw Ksiazek

Danuta Mizgalska and Jan Potempa,

Department of Microbiology, Faculty

of Biochemistry, Biophysics and

Biotechnology, Jagiellonian University,

ul. Gronostajowa 7, 30-387

Krakow, Poland

ksiazek.miroslaw@gmail.com;

dankamizgalska@gmail.com;

jan.potempa@/ouisville.edu

${ }^{t}$ These authors have contributed equally to this work and share first authorship.

Specialty section:

This article was submitted to Infectious Diseases,

a section of the journal

Frontiers in Microbiology

Received: 16 February 2015

Accepted: 29 March 2015

Published: 21 April 2015

Citation:

Ksiazek M, Mizgalska D, Eick S,

Thøgersen IB, Enghild JJ and Potempa J (2015) KLIKK proteases of Tannerella forsythia: putative virulence

factors with a unique domain

structure. Front. Microbiol. 6:312.

doi: 10.3389/fmich.2015.00312
${ }^{1}$ Department of Microbiology, Faculty of Biochemistry, Biophysics and Biotechnology, Jagiellonian University, Krakow, Poland, ${ }^{2}$ Laboratory of Oral Microbiology, Department of Periodontology, University of Bern, Bern, Switzerland, ${ }^{3}$ Department of Molecular Biology and Genetics, Center for Insoluble Protein Structures (inSPIN) and Interdisciplinary Nanoscience Center (iNANO), Aarhus University, Aarhus, Denmark, ${ }^{4}$ Department of Oral Immunology and Infectious Disease, University of Louisville School of Dentistry, Louisville, KY, USA

Comparative genomics of virulent Tannerella forsythia ATCC 43037 and a close health-associated relative, Tannerella BU063, revealed, in the latter, the absence of an entire array of genes encoding putative secretory proteases that possess a nearly identical C-terminal domain (CTD) that ends with a -Lys-Leu-lle-Lys-Lys motif. This observation suggests that these proteins, referred to as KLIKK proteases, may function as virulence factors. Re-sequencing of the loci of the $K L I K K$ proteases found only six genes grouped in two clusters. All six genes were expressed by T. forsythia in routine culture conditions, although at different levels. More importantly, a transcript of each gene was detected in gingival crevicular fluid (GCF) from periodontitis sites infected with $T$. forsythia indicating that the proteases are expressed in vivo. In each protein, a protease domain was flanked by a unique $\mathrm{N}$-terminal profragment and a C-terminal extension ending with the CTD. Partially purified recombinant proteases showed variable levels of proteolytic activity in zymography gels and toward protein substrates, including collagen, gelatin, elastin, and casein. Taken together, these results indicate that the pathogenic strain of $T$. forsythia secretes active proteases capable of degrading an array of host proteins, which likely represents an important pathogenic feature of this bacterium.

Keywords: infectious disease, periodontal disease, Tannerella forsythia, proteases, virulence

\section{Introduction}

Tannerella forsythia is a Gram-negative, asaccharolytic bacterium residing in the oral cavity. Its primary niche is a subgingival space below the gum line where, together with a diverse community of microorganism, it is a part of subgingival bacterial plaque (Colombo et al., 2009). The plaque becomes pathogenic when $T$. forsythia is joined by Porphyromonas gingivalis and Treponema denticola, grouped together into the so-called "red complex" of oral bacteria. Through release of various virulence factors, these bacteria disturb homeostasis in the periodontium leading to a sustained host inflammatory response, which erodes tooth-supporting tissues (Schenkein, 2006). Disease progression is manifested by attachment loss, alveolar bone resorption, and formation of deep

Abbreviations: SP, signal peptide; NTP, N-terminal profragment; CD, catalytic domain; CTE, C-terminal extension; CTD, C-terminal domain. 
periodontal pockets. Severe cases of periodontitis, which inflict $7-15 \%$ of the human population, may eventually result in tooth loss, if untreated (Socransky et al., 1998; Tanner and Izard, 2006; Colombo et al., 2009; Montagner et al., 2012). In addition, periodontal infection is associated with systemic diseases such as cardiovascular disease, rheumatoid arthritis, and diabetes (Suzuki et al., 2010; Lalla and Papapanou, 2011; Jeftha and Holmes, 2013; Koziel et al., 2014).

The common feature of the red complex bacteria is secretion of proteolytic enzymes, which are established virulence factors of P. gingivalis and T. denticola (Imamura et al., 2003; Ishihara, 2010). P. gingivalis proteases are especially well-investigated with respect to their structure and housekeeping functions, as well as their role in deterring host defenses and fuelling local inflammation (Guo et al., 2010). By stark contrast, very little is known regarding the T. forsythia proteases (Saito et al., 1997; Sharma, 2010), and only three enzymes have been characterized to date. The cysteine protease $\mathrm{PrtH}$ possesses a predicted caspase-like fold and detaches adherent cells from the substratum and stimulates IL-8 expression (Nakajima et al., 2006; Tomi et al., 2008; Pei and Grishin, 2009). PrtH levels are correlated with periodontal attachment loss (Hamlet et al., 2008). A second protease, karilysin, is structurally related to human matrix metalloproteases (Karim et al., 2010; Cerdà-Costa et al., 2011; Guevara et al., 2013). The enzyme may contribute to $T$. forsythia virulence by shedding of soluble, fully active tumor necrosis factor $\alpha(\mathrm{TNF} \alpha)$ from the macrophage surface (Bryzek et al., 2014), inhibition of all pathways of the complement system (Jusko et al., 2012), and degradation of the antimicrobial peptide LL-37 (Koziel et al., 2010), a component of innate immunity essential for periodontium homeostasis (Eick et al., 2014). The third protease is mirolase, a calcium-dependent serine protease with a unique mechanism of activation that may contribute to $T$. forsythia virulence by hydrolysis of human fibrinogen, hemoglobin, and LL-37 (Ksiazek et al., 2015a).

T. forsythia karilysin is a unique multi-domain protein encompassing a typical N-terminal signal peptide (SP), followed by a short propeptide conferring latency on the secreted protease with the fold closely resembling an animal matrix metalloproteinaselike catalytic domain (CD), and a C-terminal extension (CTE) (Karim et al., 2010; Cerdà-Costa et al., 2011; Lopez-Pelegrin et al., 2015). The most C-terminal part of the CTE shares sequence similarity with a conserved CTD that serves as a signal to translocate secreted proteins across the bacterial outer membrane via a novel type 9 secretion system (T9SS) first described in $P$. gingivalis (Sato et al., 2005; Nguyen et al., 2007, 2009). This system also operates in T. forsythia and another periodontal pathogen, Prevotella intermedia, mediating secretion of major virulence factors in all these pathogenic species. These virulence factors include gingipains of $P$. gingivalis, interpain of $P$. intermedia, and surface layer proteins as well as the leucine-rich protein BspA of T. forsythia (Sato et al., 2013; Zhou et al., 2013; Narita et al., 2014; Tomek et al., 2014).

The primary structure of the karilysin-derived CTD deviates from that of the classical CTD; nevertheless, karilysin secretion is dependent on T9SS (Narita et al., 2014). A blast analysis of a putative $T$. forsythia transcriptome revealed the presence of nine proteins with a KLIKK CTD, as in karilysin. Eight of these putative proteins contain a protease domain; thus we refer to them as the KLIKK proteases. They are clustered in three loci in T. forsythia ATCC 43037 but are absent in the periodontal health-associated Tannerella BU063 (Oral Taxon 286) (Beall et al., 2014). This observation suggests the KLIKK proteases may be important virulence factors. Therefore, to verify the presence, structure, expression, and activity of these putative proteases, we re-sequenced a part of the $T$. forsythia genome, determined the level of expression of the KLIKK proteases in vitro and in vivo, and characterized their proteolytic activity.

\section{Materials and Methods}

\section{Chemicals and Reagents}

The restriction endonucleases BamHI and XhoI, T4 DNA ligase, dNTPs, GeneJET ${ }^{\mathrm{TM}}$ Gel Extraction Kit, GeneJET ${ }^{\mathrm{TM}}$ PCR Purification Kit, and GeneJET ${ }^{\mathrm{TM}}$ Plasmid Miniprep Kit were purchased from Thermo Scientific Fermentas (Vilnius, Lithuania). Phusion DNA Polymerase was obtained from Thermo Scientific Finnzyme (Woburn, MA, USA). The QuikChange Lightning Site-Directed Mutagenesis Kit was obtained from Stratagene (La Jolla, CA, USA). All primers used in the study were synthesized by Genomed and "Pracownia Sekwencjonowania DNA i Syntezy Oligonukleotydów” IBB PAN (Warsaw, Poland). The expression vector pGEX-6P-1, glutathione-Sepharose 4 Fast Flow and 3C protease (PreScission) were purchased from GE Healthcare Life Sciences (Little Chalfont, UK). FTC-casein and Protein Concentrators (9K MWCO, $7 \mathrm{~mL}$ ) were obtained from Pierce Thermo Fisher Scientific (Rockford, IL). Azocoll was purchased from Calbiochem Merck (Darmstadt, Germany), DQ-gelatin was purchased from Life Technologies Thermo Fisher Scientific (Rockford, IL USA), and Elastin Congo Red was purchased from Sigma (St. Louis, MO). The molecular weight marker: "LMW" (molecular mass range: $14-97 \mathrm{kDa}$ ) was purchased from GE Healthcare. Unless otherwise indicated, all other chemicals were obtained from BioShop Canada (Burlington, ON, Canada).

\section{Re-Sequencing of Loci Encoding Putative Proteases with the KLIKK Ending}

PCR was performed using a CFX96 Touch machine (Bio-Rad Life Science Research, Hercules, CA). Each reaction consisted of $10 \mu \mathrm{l}$ of $5 \times$ Phusion HF Buffer, $5 \mu$ l of $2 \mathrm{mM}$ dNTPs, $5 \mu l$ of specific primer mix, as listed in Supplementary Table 1 (final primer concentration, $0.5 \mu \mathrm{M}$ ), $1.5 \mu \mathrm{l}$ of DMSO, $2.5 \mu \mathrm{l}$ (50 ng) of genomic DNA isolated from T. forsythia strain ATCC 43037, $1 \mu$ l of Phusion DNA Polymerase, and water to a final volume of $50 \mu \mathrm{l}$. The PCR reaction consisted of an initial denaturation step at $98^{\circ} \mathrm{C}$ for $2 \mathrm{~min}$, followed by 35 cycles of $10 \mathrm{~s}$ at $98^{\circ} \mathrm{C}, 30 \mathrm{~s}$ at a primer specific annealing temperature (Supplementary Table 1), and $30 \mathrm{~s}$ per $1 \mathrm{~kb}$ of predicted PCR product at $72^{\circ} \mathrm{C}$, and a final extension step $\left(10 \mathrm{~min}\right.$ at $\left.72^{\circ} \mathrm{C}\right)$. Amplicons were separated by electrophoresis in a $1 \%$ agarose gel, cut from the gel, and purified using the GeneJET $^{\mathrm{TM}}$ Gel Extraction Kit. The purified PCR products were sent for DNA sequencing using specific primers (Supplementary Table 1). 


\section{Molecular Cloning}

Genomic DNA was isolated from T. forsythia strain ATCC 43037 using the Genomic Mini System (A\&A Biotechnology, Gdansk, Poland), according to the manufacturer's recommendations. The entire genes encoding forsilysin (BFO_1168), miropsin-1 (BFO_1179), mirolysin (BFO_2661), and miropsin2 (BFO_2679), except for the nucleotide sequences encoding the predicted SPs, were amplified by PCR, purified, and cloned into the pGEX-6P-1 expression vector using BamHI/XhoI and BamHI/EcoRI sites, and specific PCR primers (Supplementary Table 1). The plasmids encoding karilysin and mirolase were obtained as described previously (Karim et al., 2010; Ksiazek et al., 2015a). The resulting recombinant products include an N-terminal GST tag and a PreScission protease cleavage site, followed by the protein sequence. All plasmids were verified by DNA sequencing.

\section{Real-Time PCR}

T. forsythia RNA was isolated from 5-day-old plates using an innuPREP RNA Mini Kit (Analytic Jena, Jena, Germany). Before cDNA synthesis, the RNA was digested with RQ1 DNase (Promega, Madison, WI, USA) and purified using the TRI Reagent (Ambion, Life Technologies). RNA (1.6 $\mu \mathrm{g})$ was then reverse transcribed with cDNA High Capacity cDNA Reverse Transcription Kit (Applied Biosystems, Life Technologies). The real-time PCR was performed on a CFX96 Touch machine. A single reaction consisted of $7.5 \mu$ l of FastStart Essential DNA Green Master mix (Roche, Basel, Switzerland), $1 \mu l$ of $300 \mathrm{nM}$ target specific primer mix (Supplementary Table 1), $5 \mu l$ of cDNA (diluted 1:10), and $1.5 \mu \mathrm{l}$ of water. The PCR reaction consisted of an initial denaturation step at $95^{\circ} \mathrm{C}$ for $10 \mathrm{~min}$, followed by 40 cycles of $10 \mathrm{~s}$ at $95^{\circ} \mathrm{C}, 30 \mathrm{~s}$ at a primer specific annealing temperature, and $30 \mathrm{~s}$ at $72^{\circ} \mathrm{C}$. All samples were analyzed in triplicate. Relative transcripts levels were calculated using the modified $\Delta \Delta \mathrm{Ct}$ method (Pfaffl, 2001).

\section{Determination of the Expression of the KLIKK Proteases In Vivo}

Patients with diagnosed chronic periodontitis attending the Clinic of Periodontology at the University Hospital of Jena were recruited for this study. For detection of $T$. forsythia in clinical samples, GCF samples were obtained from patients with severe periodontitis [aggressive periodontitis $(n=17)$ and chronic periodontitis $(n=37)$ ] and from six healthy controls. Two paper points were inserted in each pocket for $20 \mathrm{~s}$, and DNA was subsequently extracted using the Genomic Mini System, according to the manufacturer's recommendations. PCR for detection of T. forsythia was carried out as described previously (Ashimoto et al., 1996). To determine whether KLIKK protease genes were transcribed in vivo, an aliquot of GCF was stored at $-20^{\circ} \mathrm{C}$ until mRNA was extracted for RT-PCR analysis. Total RNA from $\sim 50 \mu \mathrm{l}$ of GCF was purified using an RNeasy kit (Qiagen, Venlo, Limburg, Netherlands), and cDNA was synthesized from $1 \mu \mathrm{g}$ of total RNA employing the Omniscript kit (Qiagen). Oligonucleotide primers (Supplementary Table 1) were used at a final concentration of $0.5 \mu \mathrm{M}$. The PCR with Taq polymerase was performed for 30 cycles, consisting of denaturation at $94^{\circ} \mathrm{C}$ for $30 \mathrm{~s}$, annealing at $56^{\circ} \mathrm{C}$ for $25 \mathrm{~s}$, and polymerization at $72^{\circ} \mathrm{C}$ for $30 \mathrm{~s}$. The amplified PCR products were then analyzed by electrophoresis in a $2 \%$ agarose gel.

\section{Expression and Purification of Recombinant Proteins}

The plasmids encoding recombinant $T$. forsythia KLIKK proteases were transformed into Escherichia coli strain BL21 (DE3) (New England Biolabs, Ipswich, MA) under the control of the T7 promoter. Transformed E. coli hosts were grown in LB medium at $37^{\circ} \mathrm{C}$ to an $\mathrm{OD}_{600}$ ranging from 0.75 to 1 and cooled for $30 \mathrm{~min}$ at $4^{\circ} \mathrm{C}$, and expression of recombinant proteins was induced by the addition of $0.25 \mathrm{mM}$ isopropyl-1-thio- $\beta$-D-galactopyranoside (IPTG). After culture for $6 \mathrm{~h}$ at $20^{\circ} \mathrm{C}$, cells were harvested by centrifugation $\left(15 \mathrm{~min}, 6000 \times \mathrm{g}, 4^{\circ} \mathrm{C}\right)$, re-suspended in PBS $(15 \mathrm{ml}$ per pellet from $1 \mathrm{~L}$ of culture), and subsequently lysed by sonication (cycle of $30 \times 0.5 \mathrm{~s}$ pulses at a power output of $70 \%$ per pellet from $1 \mathrm{~L}$ of culture) using a Branson Sonifier Digital 450 (Branson Ultrasonics, Danbury, CT, USA). The cell lysates were clarified by centrifugation $\left(40 \mathrm{~min}, 40,000 \times \mathrm{g}, 4^{\circ} \mathrm{C}\right.$ ), filtered through a $0.45 \mathrm{~mm}$ syringe filter, and loaded onto a glutathione-Sepharose 4 Fast Flow column (bed volume, $5 \mathrm{ml}$ ) equilibrated with PBS at $4{ }^{\circ} \mathrm{C}$. Recombinant proteins were eluted using $50 \mathrm{mM}$ Tris$\mathrm{HCl}, \mathrm{pH}$ 8.0, supplemented with $10 \mathrm{mM}$ reduced glutathione. Alternatively, $10 \mathrm{ml}$ of PBS containing $100 \mu \mathrm{l}$ of PreScission protease stock solution $\left(1 \mathrm{U} \mathrm{ml}^{-1}\right)$ was applied to the column and incubated for $40 \mathrm{~h}$ at $4^{\circ} \mathrm{C}$. Protein concentration was determined by measurement of absorbance at $280 \mathrm{~nm}$ using a Nanodrop spectrophotometer (NanoDrop products, Wilmington, DE, USA). The purity of the proteins was verified by SDS-PAGE electrophoresis using 10\% gels (acrylamide/bis-acrylamide ratio, 33:1) and the Tris-HCl/Tricine buffer system (Schägger and von Jagow, 1987). Gels were stained with 0.1\% Coomassie Brilliant Blue R-250 in 10\% acetic acid and destained in 30\% methanol, $10 \%$ acetic acid, and $1 \%$ acetic acid.

\section{Zymography}

Zymographic analysis was performed on the purified KLIKK proteases, mixed 1:1 with sample buffer $(0.125 \mathrm{M}$ Tris- $\mathrm{HCl}, \mathrm{pH} 7.8$, $20 \%$ glycerol, $4 \%$ SDS, and $0.1 \%$ Bromophenol Blue) for $15 \mathrm{~min}$ at $20^{\circ} \mathrm{C}$, and then electrophoretically resolved in $12 \%$ SDS-PAGE gels (acrylamide/bis-acrylamide ratio, 27.5:1) containing casein or gelatin at a final concentration of $0.1 \mathrm{mg} \mathrm{ml}^{-1}$. The gels were washed twice in $2.5 \%$ Triton X-100 for $30 \mathrm{~min}$, followed by incubation in developing buffer $(0.2 \mathrm{M}$ Tris- $\mathrm{HCl}, \mathrm{pH} 7.8,5 \mathrm{mM}$ $\mathrm{CaCl}_{2}$, and $1 \mathrm{mM} \mathrm{DTT}$ ) for $3 \mathrm{~h}$ at $37^{\circ} \mathrm{C}$. Finally, gels were incubated in destaining/fixing solution [methanol:acetic acid:water $(30: 10: 60)]$, then stained with $0.1 \%$ amido black in $10 \%$ acetic acid for $1 \mathrm{~h}$ and destained successively in destaining/fixing solution, $10 \%$ acetic acid, and $1 \%$ acetic acid, which revealed clear zones of substrate hydrolysis on a blue background.

\section{Proteolytic Activity Assay}

To determine the activity of the proteases against Azocoll and Elastin Congo Red, $2.5 \mu \mathrm{g}$ of each KLIKK protease was diluted in assay buffer (50 mM Tris, $\mathrm{pH} 8.0,2.5 \mathrm{mM} \mathrm{CaCl}_{2}$, and $0.02 \%$ $\mathrm{NaN}_{3}$ ) to a final volume of $125 \mu \mathrm{l}$, and then mixed with $125 \mu \mathrm{l}$ of 
substrate in assay buffer $\left(15 \mathrm{mg} \mathrm{ml}^{-1}\right)$, followed by incubation for $2 \mathrm{~h}$ (Azocoll) or $16 \mathrm{~h}$ (Elastin Congo Red) at $37^{\circ} \mathrm{C}$ with shaking. For Elastin Congo Red, human neutrophil elastase (BioCentrum, Krakow, Poland) was used as a positive control. Undigested substrate was removed by centrifugation $(5 \mathrm{~min}, 16,100 \times$ $\mathrm{g}$ ), and the absorbance of the supernatant at $520 \mathrm{~nm}$ (Azocoll) or $495 \mathrm{~nm}$ (Elastin Congo Red) was measured using a SpectraMAX microplate reader (Molecular Devices, Sunnyvale, CA). For FTC-casein and DQ-gelatin, mixtures containing $2.5 \mu \mathrm{g}$ of each KLIKK protease in $100 \mu \mathrm{l}$ of assay buffer were prepared directly in the wells of black microtiter plates (Nunc, Roskilde, Denmark). Next, $100 \mu \mathrm{l}$ of substrate solution in assay buffer (50 and $100 \mu \mathrm{g}$ $\mathrm{ml}^{-1}$ for FTC-casein and DQ-gelatin, respectively) was added, and the rate of substrate hydrolysis was recorded as the increase in fluorescence $\left(\lambda_{\mathrm{ex}}=385 \mathrm{~nm}, \lambda_{\mathrm{em}}=438 \mathrm{~nm}\right.$ for FTC-casein, and $\lambda_{\mathrm{ex}}=495 \mathrm{~nm}, \lambda_{\mathrm{em}}=515 \mathrm{~nm}$ for DQ-gelatin) using a fluorescence microplate reader (SpectraMaxGmini XS, Molecular Devices).

\section{Results}

\section{Re-Sequencing of Fragments of T. forsythia Genome Containing KLIKK Proteases}

The sequence of karilysin and mirolase available in the database (GenBank database, accession number: CP003191) was previously shown to be incorrect (Karim et al., 2010; Ksiazek et al., 2015a). Thus, we re-sequenced three fragments of the T. forsythia genome containing the eight KLIKK protease loci (Figure 1A). Amplification of the desired regions was performed with primers nested in genes flanking the protease open reading frames (ORFs). Six KLIKK proteases were found: three serine proteases (BFO_1179, miropsin-1; BFO_2665, mirolase; BFO_2679, miropsin-2) and three metalloproteases ( $B F O_{-} 1168$, forsilysin; BFO_2661, mirolysin; BFO_2683, karilysin) (Figure 1B). The sequencing analysis revealed that the available genomic sequence of $T$. forsythia contains a number of errors, including the presence of non-existing ORFs (BFO_0703 and BFO_2675), errors in the prediction of ORF N-termini (forsilysin, mirolase), and differences in amino acid sequences within ORFs resulting from point mutations, deletions, and insertions. It should be noted that only the database sequence for mirolysin is correct.

To exclude the possibility that the two ORFs (BFO_0703 and $\left.B F O \_2675\right)$ we did not find within the analyzed fragments of the $T$. forsythia genome were present at other loci, we tried to amplify these two genes (using primers designed on the sequences in the database), but the results were negative in both cases, and we failed to obtain the desired PCR products.

All the KLIKK proteases were preceded by ORFs encoding small (360-550 bp) putative proteins, predicted to be lipoproteins of unknown function (Figure 1B). Alignment of amino acid and nucleic acid sequences of these six ORFs revealed that they share no significant sequence similarity. These ORFs possess a common feature: at the end of each predicted lipoprotein there is a $\sim 50 \mathrm{bp}$ long AT rich tract, but without any consensus sequence (data not shown).

\section{Multi-Domain Structure of the KLIKK Proteases}

The alignment of the KLIKK proteases revealed the multi-domain structure of the analyzed enzymes (Figure 2).
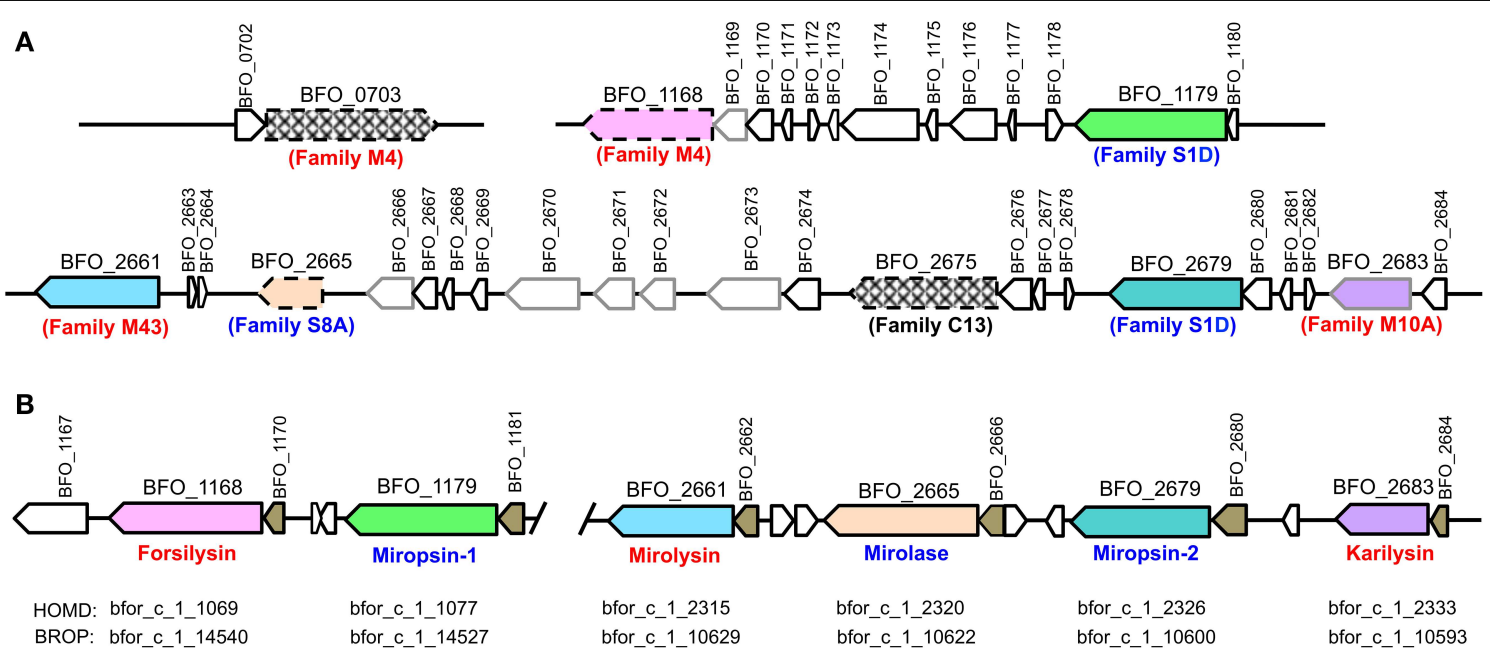

FIGURE 1 | Gene arrangement of $T$. forsythia ATCC 43037 KLIKK proteases. (A) A GenBank (accession number: CP003191, http://www.ncbi. $\mathrm{nlm}$.nih.gov/genbank) deposited sequence, prior to re-sequencing. Arrows framed by a gray line-ORFs identified as pseudogenes; hatched and color arrows framed with a broken line - protease genes absent in the genome and with errors in the prediction of ORF N-termini, respectively. (B) Re-sequenced and corrected gene arrangement. Arrow boxes: black, genes; gray, pseudogenes; color filled, genes encoding proteases; and brown, genes encoding putative lipoproteins. Designations of the peptidase family
( $M$, metallopeptidase; S, serine peptidase; and C, cysteine peptidase) of putative proteases are indicated below the arrows on (A). Names of the proteases characterized with respect to expression by T. forsythia and proteolytic activity is shown in (B). Red, metalloproteases; blue, serine proteases. HOMD: Human Oral Microbiome Taxon Description, Tannerella forsythia strain 92A2 (HOMD, http://www.homd.org); BROP: Bioinformatics Resource for Oral Pathogens, Tannerella forsythia 92A2 (BROP, http://www. brop.org). These sequence data have been submitted to the GenBank database under accession numbers KP715368 and KP715369. 
A

FIGURE 2 | Predicted multi-domain structure of $T$. forsythia KLIKK proteases. (A) The corrected sequences of $T$. forsythia proteases were aligned using ClustalW (http://www.ebi.ac.uk/Tools/msa/clustalw2/). Based on this, the following structure of KLIKK proteases, beginning from the N-terminus, was proposed: SP (gray box), NTP (white box), $\mathrm{CD}$ (color boxes) containing all amino acids directly involved in proteolysis (in black), and CTE containing the variable region $B$ flanked by two conserved regions, 30 amino acids $A$, and the last 86 amino acids (CTD). The catalytic amino acids and protease family (in brackets next to the gene name) were predicted using the MEROPS database. The signal peptides were predicted using the SignalP 3.0 Server (Bendtsen et al., 2004). (B) Alignment of region A from KLIKK proteases. The identical amino acids in at least four proteases are highlighted in gray, and the asterisks indicate conserved amino acids in all proteases. (C) Consensus sequence of CTD from KLIKK proteases. Low case fonts indicate residues occurring only once at the specific position. The amino acids conserved in known CTDs of proteins of $P$. gingivalis and $T$. forsythia is marked in red.

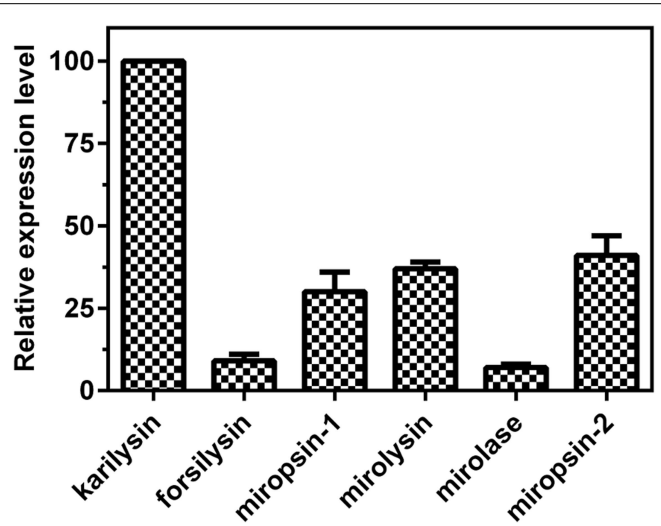

FIGURE 3 | The expression level of KLIKK proteases found in the genome of $\boldsymbol{T}$. forsythia. The expression level of six proteases was determined by real-time PCR. The transcript level of karilysin was arbitrarily set as 100 . The results presented are mean \pm SD from three experiments.

Beginning from the N-terminus, the KLIKK proteases consist of a classical SP (predicted using SignalP 3.0 Server, Bendtsen et al., 2004), an N-terminal profragment (NTP), a CD containing all the amino acid residues crucial for proteolytic activity (MEROPS database, Rawlings et al., 2014), and a CTE. The CTE is unique for each protease and consists of a sequence of 140-160 residues flanked by two conserved regions: a motif of 30 residues at the beginning and a domain of 86 residues at the end. The variable region of the CTE was identical only for the two S1D serine proteases, miropsin-1 and miropsin-2. Interestingly, with the exception of the CTD domain, which shares some similarity with classical CTDs found in proteins of $P$. gingivalis and T. forsythia, e.g., gingipains and $T$ forsythia S-layer proteins, respectively, other segments of the KLIKK proteases flanking the protease domains do not possess significant homology to any known proteins (Nguyen et al., 2007).

\section{Expression Level and Prevalence of KLIKK Proteases}

Given that not every putative ORF found in the sequenced genome is expressed, we performed real-time PCR to determine the expression levels of KLIKK proteases in in vitro culture of a laboratory strain of T. forsythia ATCC 43037 (Figure 3). We were able to detect transcripts for each KLIKK protease. Karilysin exhibited the highest expression level, while expression of the serine proteases miropsin-1, mirolase, and miropsin-2 was 3-fold lower, and expression of the two metalloproteases, forsilysin and mirolysin, was 10-fold lower than that of karilysin.

In prokaryotes, there is a well-described phenomenon of negative selection leading to the removal of any unnecessary genes (Burke and Moran, 2011). Therefore, we determined the 
expression of the KLIKK proteases in vivo in the GCF collected from $T$. forsythia positive patients suffering from different forms of periodontitis. Transcripts of all the KLIKK proteases were found in the majority of samples testing positive for the presence of T. forsythia (Table 1).

\section{Proteolytic Activity of the KLIKK Proteases}

To confirm that the KLIKK proteases are active proteolytic enzymes, we expressed each of them as fusion proteins with an $\mathrm{N}$-terminal glutathione-S-transferase (GST). The proteases were purified by affinity chromatography on glutathione-Sepharose

TABLE 1 | Prevalence of KLIKK protease transcripts in GCF from patients suffering from periodontitis.

\begin{tabular}{lccc}
\hline Primers for: & Patients with chronic periodontitis: $\mathbf{3 7}$ samples & Patients with aggressive periodontitis: $\mathbf{1 7}$ samples & Healthy controls: $\mathbf{6}$ samples \\
\hline T. forsythia & $28 / 37(76 \%)$ & $15 / 17(88 \%)$ & $1 / 6(17 \%)$ \\
forsilysin & $26 / 28(93 \%)$ & $11 / 15(73 \%)$ & $0 / 1(0 \%)$ \\
miropsin-1 & $22 / 28(79 \%)$ & $9 / 15(60 \%)$ & $1 / 1(0 \%)$ \\
mirolysin* & $24 / 28(86 \%)$ & $13 / 15(87 \%)$ & $0 / 1(0 \%)$ \\
mirolase & $27 / 28(96 \%)$ & $15 / 15(100 \%)$ & $1 / 1(100 \%)$ \\
miropsin-2 & $28 / 28(100 \%)$ & $15 / 15(100 \%)$ & $1 / 1(100 \%)$ \\
karilysin* & $27 / 28(96 \%)$ & $14 / 15(93 \%)$ & $1 / 1(100 \%)$
\end{tabular}

${ }^{\star}$ Results for karilysin and mirolysin were published previously (Jusko et al., 2012, under review).

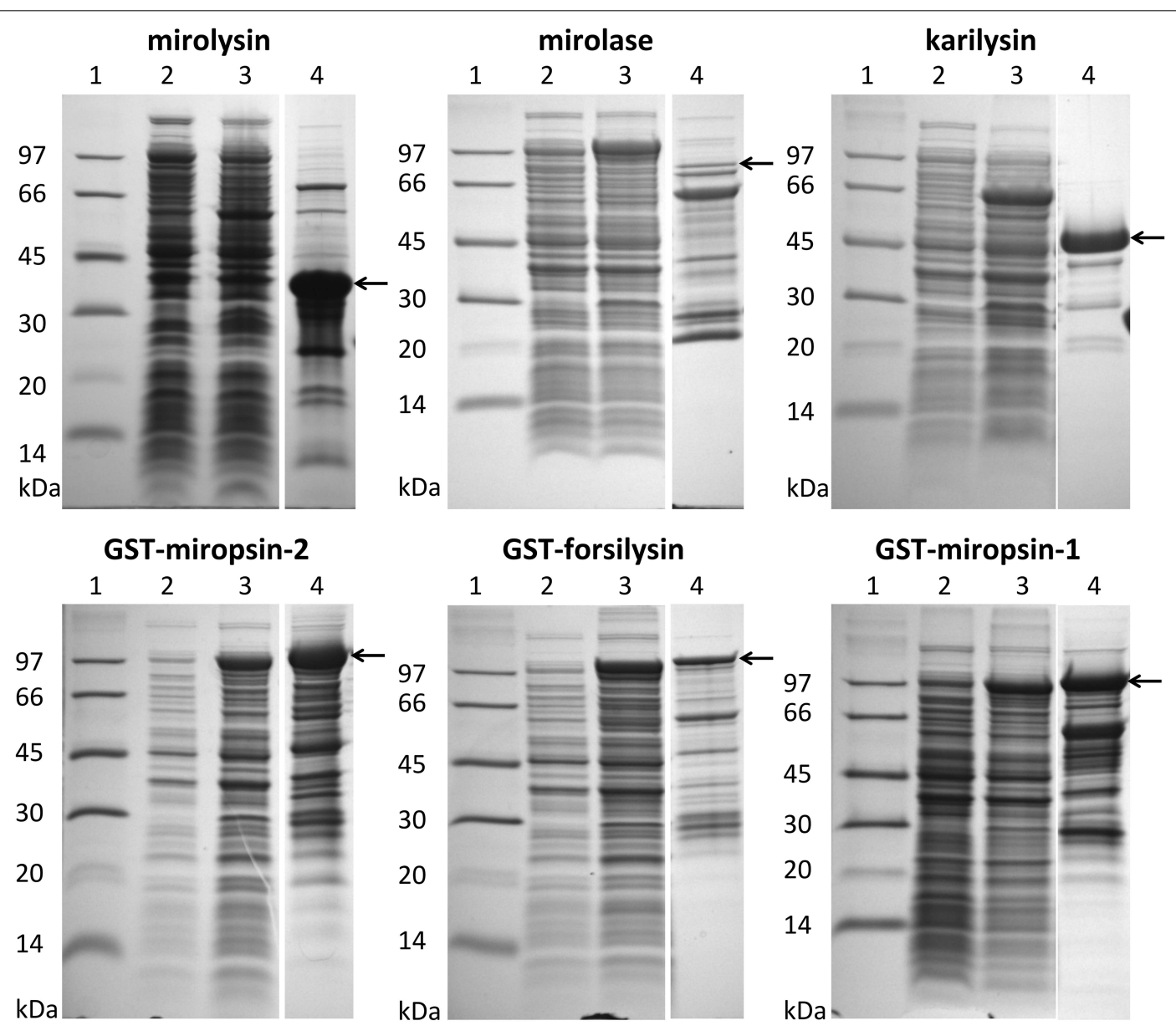

FIGURE 4 | Expression and purification of KLIKK proteases.

Escherichia coli extracts and fusion proteins or tag-free proteases after purification on glutathione-Sepharose and, if applicable, in column digestion with PreScission protease, were resolved by SDS-PAGE. Lane 1, protein molecular weight marker "LMW." Lane 2, E. coli extracts before IPTG induction. Lane 3, E. coli extracts at $6 \mathrm{~h}$ after protein expression stimulation with IPTG. Lane 4, purified proteases as tag-free proteolytic enzymes or fusion proteins with GST. The arrows indicate the band corresponding to the theoretical molecular mass of the purified protein. 


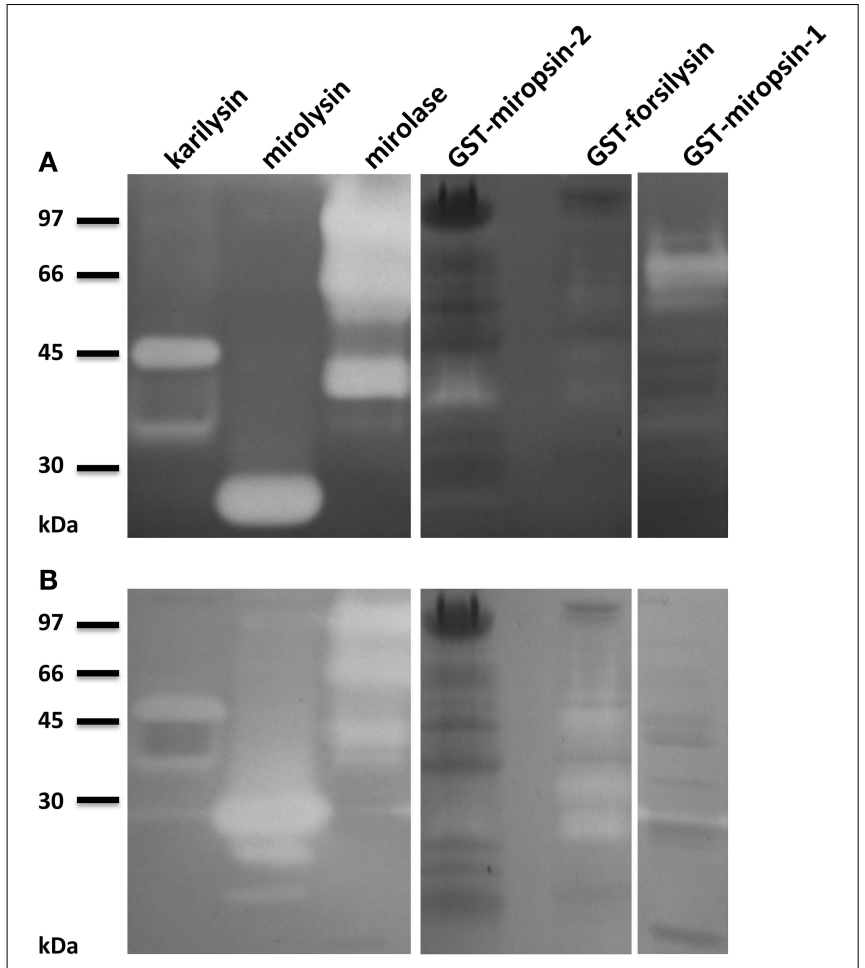

FIGURE 5 | Zymography analysis of KLIKK proteases. The activity of six proteases (karilysin $(2.5 \mu \mathrm{g})$, mirolysin $(2.5 \mu \mathrm{g})$, mirolase $(2.5 \mu \mathrm{g})$, GST-miropsin-2 $(15 \mu \mathrm{g})$, GST-forsilysin $(5 \mu \mathrm{g})$, and GST-miropsin-1 $(7.5 \mu \mathrm{g})$ ) was analyzed by zymography using casein (A) and gelatin (B) as a substrate.

(Figure 4). For the recombinant mirolysin, mirolase, and karilysin, the GST was removed by cleavage with the PreScission protease. The activities of miropsin-1, miropsin-2, and forsilysin were tested as fusion proteins due to their instability without the GST tag. In the case of mirolysin, despite using different E. coli strains and screening many expression conditions, we were unable to obtain a sufficient amount of the full-length enzyme. For this reason, we expressed this protease without the CTE region, but with the NTP, which could act as a chaperone and, thus, be required for proper folding of the enzyme (Bryan, 2002) (Figure 2). The recombinant proteins were used in further experiments, as described below.

First, activity of the purified KLIKK proteases was tested in zymography with two substrates, gelatin, and casein (Figure 5). All investigated proteases were active against at least one substrate. Moreover, in all cases, with the exception of miropsin-2, there was more than one band of activity visible. This observation indicates that the KLIKK proteases, similarly to karilysin, undergo auto-processing into lower molecular mass forms.

Finding a synthetic substrate for every novel protease paves the way for further research, so we checked whether the KLIKK proteases are active against the commercially available labeled protein substrates Azocoll, DQ-gelatin, Elastin Congo Red, and FTC-casein (Figure 6). For three proteases, forsilysin, mirolysin, and mirolase, we identified at least one substrate that was digested with efficiency comparable to that of karilysin. Although the activity of the two remaining proteases, miropsin-1 and miropsin-2, were several fold lower than karilysin, we were able to identify substrates that could be used for monitoring the proteolytic activity. The low activity is most likely due to enzyme latency imposed by the NTP and/or a low level of auto-activation in the presence of the GST tag. The most active enzyme against the investigated substrates was mirolysin. Interestingly, three proteases, karilysin, forsilysin, and miropsin-2, were able to digest Elastin Congo Red. Elastin is one of the major components of the connective tissue. Since miropsin-1 and miropsin-2 exhibited low activity to the protein substrates, we examined whether these two serine proteases were active against several synthetic amino acid chromogenic substrates with p-nitroaniline ( $p \mathrm{NA}$ ) as a leaving group including MeoSuc-AAV- $p$ NA, Suc-AAPF$p N A$, Suc-AAPL- $p$ NA, Suc-AAPA- $p$ NA, p-Tosyl-GPK- $p$ NA, and Suc-AAPR-pNA. Miropsin-2 was active against one substrate, Suc-AAPL- $p$ NA and the activity was $15 \mathrm{mAbs}_{410} \mu \mathrm{g}^{-1} \mathrm{~h}^{-1}$.

\section{Discussion}

An annotated T. forsythia genome is currently available (GenBank database, accession number: CP003191), but initial attempts to amplify some of the genes encoding the KLIKK proteases failed. Also a previous attempt to amplify the karilysin gene failed due to an incorrect sequence in the database (Karim et al., 2010). Therefore, we re-sequenced parts of the genome encompassing genes encoding the putative KLIKK proteases and found that the available genomic sequence contained a number of errors. The sequences of the $T$. forsythia genome fragments encoding the KLIKK proteases may have generated problems in the correct assembly of the genome due to the high A/T content, the presence of $\mathrm{A} / \mathrm{T}$ tracks, and the identical sequential motifs shared by the KLIKK proteases (Schatz et al., 2010).

The corrected sequences revealed that each KLIKK protease gene is preceded by a $50 \mathrm{bp} \mathrm{A/T}$ rich tract, which is probably responsible for the initiation of protein translation through a non-classical, Shine-Dalgarno sequence independent mechanism. Similar phenomena were described for other members of the Bacteroidetes (Accetto and Avguštin, 2011), as well as E. coli (Skorski et al., 2006; Nakagawa et al., 2010). The non-classical mechanism depends on the presence of a secondary structure of low stability $\left(\Delta \mathrm{G}>-2.1 \mathrm{kcal} \mathrm{mol}^{-1}\right)$ in a $5 / \mathrm{UTR}$ region adjacent to a start codon (Accetto and Avguštin, 2011). The analysis of a 30 bp long 5/UTR region of KLIKK proteases by mfold RNA (Zuker, 2003) revealed the presence of very weak secondary structures $\left(\Delta \mathrm{G}>0.4 \mathrm{kcal} \mathrm{mol}^{-1}\right)$. Moreover, a Shine-Dalgarno sequence could not be identified, suggesting that all of the KLIKK proteases use a Shine-Dalgarno sequence independent mechanism for initiation of translation.

The bioinformatics analysis revealed that the KLIKK proteases possess a unique multi-domain structure. Briefly, the CD is flanked by the NTP and CTE domains, which do not have homology to any known proteins. The length of NTP is unique for each protease and varies from 14 (karilysin) to 225 residues (forsilysin and miropsin-1). The results obtained for karilysin suggest that the NTP region is responsible for the latency of the KLIKK proteases. Due to the irreversible nature of peptide bond hydrolysis, proteases are often synthesized as inactive zymogens 

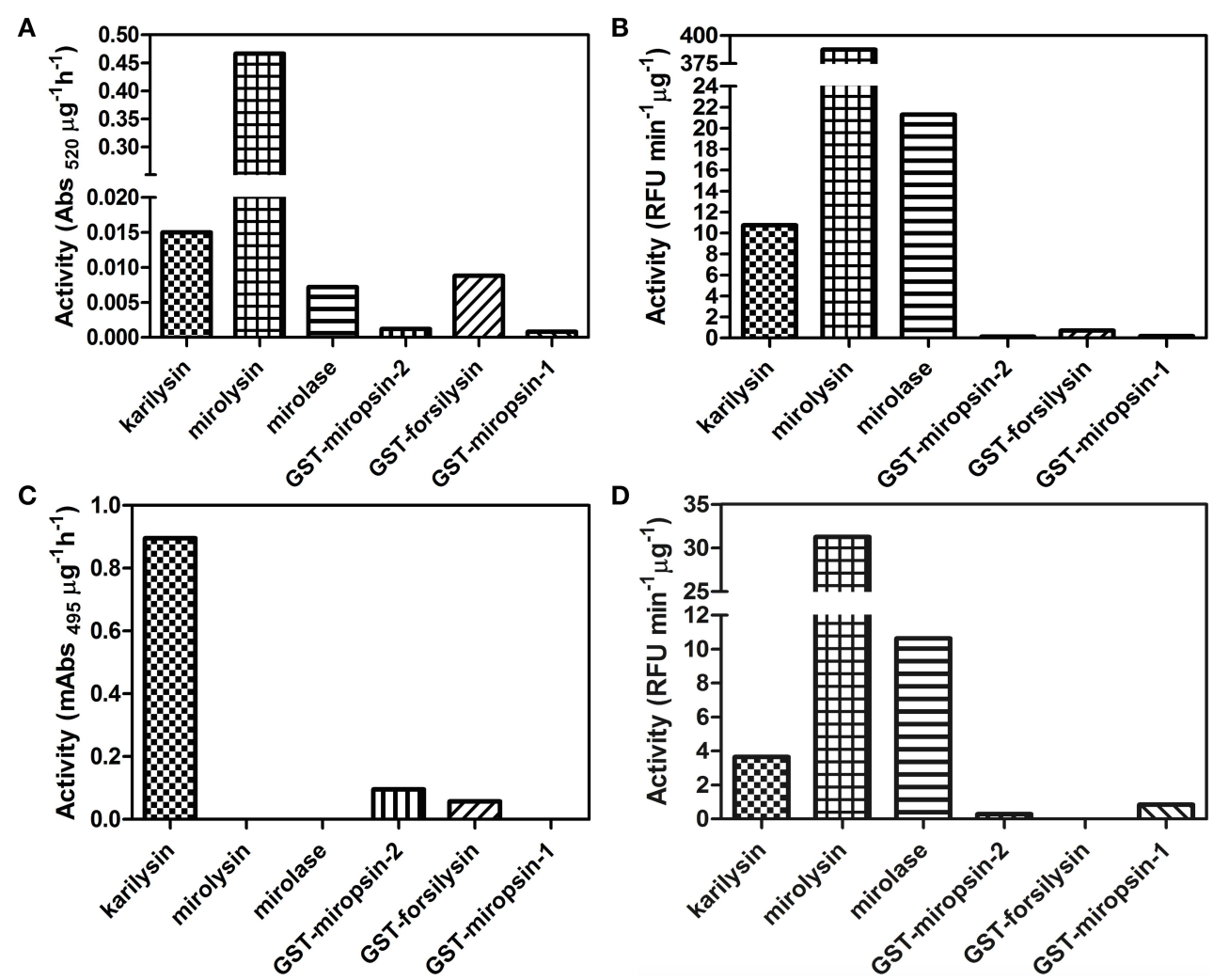

FIGURE 6 | Activity of KLIKK proteases against four protein substrates: Azocoll (A), DQ-gelatin (B), Elastin Congo Red (C), and FTC-casein (D). For Elastin Congo Red, human neutrophil elastase was used as a positive control, and its activity in the same conditions were $3.8 \mathrm{mAbs}_{495} \mu \mathrm{g}^{-1} \mathrm{~h}^{-1}$.

to prevent inappropriate proteolytic damage. The zymogenicity is frequently exerted by the NTP, which needs to be removed to generate a full active protease. In Gram-negative bacteria, this mechanism is thought to protect the periplasm against unwanted proteolytic activity (Veillard et al., 2013).

The CD is followed by a CTE, which ends in a conserved region of 86 residues with some similarity to the CTD found in many secretory proteins of $P$. gingivalis and T. forsythia (Nguyen et al., 2007; Veith et al., 2009). The CTD is a signal for secretion of proteins through a recently described type 9 secretion system (T9SS). The 22 C-terminal residues of the CTD are essential for targeting proteins to the outer membrane translocon (Shoji et al., 2011). Interestingly, among all identified proteins terminating with a CTD in T. forsythia, only a few amino acids residues within the essential region are highly conserved, PxGxYVV and KxxxK (marked in red in Figure 2). The sequence of the rest of the CTD is variable (Veith et al., 2013). Therefore, the virtually identical CTDs in all the KLIKK proteases are unusual, and may indicate a common origin of this domain, which was acquired relatively recently. Explanation of the origin and biological impact of the CTD requires further research.

By analogy to $P$. gingivalis, the presence of the CTD fragment implies that the KLIKK proteases are secreted, posttranslationally modified, and then retained on the bacterial surface. This assumption is partially confirmed by the fact that karilysin is released into the medium in the form of the fully processed $18 \mathrm{kDa}$ protease by the laboratory strain $T$. forsythia ATCC 43037 when grown under normal conditions (data not shown). However, the KLIKK proteases do not seem to be associated with the cell envelope, because none of them was found among 221 proteins identified in the cell envelope proteome of $T$. forsythia (Veith et al., 2009). By contrast, three KLIKK proteases, miropsin-2 (bfor_c_1_10600), karilysin (bfor_c_1_10593), and forsilysin (bfor_c_1_14540), were recently shown to be secreted by T9SS (Narita et al., 2014). Together, these findings fully confirmed the release of the KLIKK proteases as soluble forms into the extracellular environment.

Karilysin, the first thoroughly characterized protease of $T$. forsythia, processes itself into shorter forms resulting in formation of the mature enzyme, Kly18. This modification is accompanied by a large increase in proteolytic activity (Karim et al., 2010). Similarly, all KLIKK proteases, with the exception of miropsin2 , occur in several active forms, strongly suggesting that these KLIKK proteases also process themselves into low molecular weight forms through sequential proteolytic cleavages. Based on the results obtained for karilysin and mirolase, the final products of auto-processing of the KLIKK proteases is predicted to be CDs without the NTP or the CTE, and at least transiently accompanied by protease-resistant domains derived from the CTE (Karim et al., 2010; Ksiazek et al., 2015a). Thus, it is tempting to speculate 
that the variable region in the KLIKK proteases could have a biological function unique for each KLIKK protease. Solving the role of these domains in $T$. forsythia virulence will require further studies.

Recently, a genome of the non-pathogenic oral bacterium Tannerella BU063 (oral taxon 286), which is closely related to T. forsythia, was sequenced (Beall et al., 2014). None of the KLIKK homologs were identified in this genome. However, a BU063 predicted ORF, T229_10715 (GenBank accession number: ETK04104.1), shows a high degree of similarity to the CD of mirolase, but lacks a SP, NTP, and the variable region characteristic of the CTE in the KLIKK proteases. Interestingly, T229_10715 possesses a predicted CTD, which is significantly different from the CTD conserved in the KLIKK proteases. These findings may suggest that during evolution, virulence genes encoding proteases already present in the ancestral genome, or acquired through horizontal gene transfer (Cerdà-Costa et al., 2011), were fused to sequences coding for the SP, NTE, and CTE, thus generating the KLIKK proteases. The change in destination of the proteases from cytoplasm to extracellular environment would allow for acquisition of novel pathogenesis-related functions. Interestingly, T. forsythia also produces a protease inhibitor of the serpin superfamily, miropin (Ksiazek et al., 2015b), with the likely function of preventing proteolytic damage from secreted serine proteases. A miropin homolog (GenBank accession number: ETK05009.1) is present in Tannerella BU063, which possesses genes encoding putative serine proteases related to the T. forsythia KLIKK proteases. By stark contrast, neither the KLIKK proteases nor the miropin homologs are present in the sequenced genome of Tannerella sp. 6_1_58FAA_CT1 isolated from the human gastrointestinal tract (GenBank accession number: NZ_ACWX00000000.1). Thus, acquisition of the KLIKK proteases may be associated with the gain of virulence by $T$. forsythia.

Collectively, our data indicate that genes of the KLIKK proteases are efficiently transcribed in in vitro culture. Moreover, transcripts of all the KLIKK proteases were detected in GCF sampled from periodontitis sites infected with T. forsythia. This

\section{References}

Accetto, T., and Avguštin, G. (2011). Inability of Prevotella bryantii to form a functional Shine-Dalgarno interaction reflects unique evolution of ribosome binding sites in Bacteroidetes. PLoS ONE 6:e22914. doi: 10.1371/journal.pone.0022914

Ashimoto, A., Chen, C., Bakker, I., and Slots, J. (1996). Polymerase chain reaction detection of 8 putative periodontal pathogens in subgingival plaque of gingivitis and advanced periodontitis lesions. Oral Microbiol. Immunol. 11, 266-273. doi: 10.1111/j.1399-302X.1996.tb00180.x

Beall, C. J., Campbell, A. G., Dayeh, D. M., Griffen, A. L., Podar, M., and Leys, E. J. (2014). Single cell genomics of uncultured, health-associated Tannerella BU063 (Oral Taxon 286) and comparison to the closely related pathogen Tannerella forsythia. PLoS ONE 14:e89398. doi: 10.1371/journal.pone. 0089398

Bendtsen, J. D., Nielsen, H., von Heijne, G., and Brunak, S. (2004). Improved prediction of signal peptides: SignalP 3.0. J. Mol. Biol. 340, 783-795. doi: 10.1016/j.jmb.2004.05.028 observation suggests an active role for the KLIKK proteases in periodontal lesions. This hypothesis is corroborated by the involvement of karilysin in evasion of innate host defenses through cleavage of LL-37 (Koziel et al., 2010) and inactivation of the complement system by karilysin and mirolysin (Jusko et al., 2012; Jusko, unpublished). However, the elastin degradation by three of the KLIKK proteases described here may also contribute to the connective tissue damage at the infected periodontal site. Although the exact biological functions of the KLIKK proteases still requires further research (Van Damme et al., 2008), they do now appear to be potent and versatile virulence factors of $T$. forsythia.

\section{Author Contributions}

MK, DM, SE, IT designed and conceived experiments; MK, DM, SE, IT performed the experiments; MK, DM, SE, JE, JP analyzed data; MK, DM and JP wrote the paper.

\section{Acknowledgments}

This work was supported in part by grants from the National Science Center (NCN, Krakow, Poland); 2013/08/T/NZ1/00315 (a doctoral scholarship to MK) and 2012/04/A/NZ1/00051, NIH/NIDCR (DE 09761 and DE 022597), the European Commission (FP7-PEOPLE-2011-ITN-290246 "RAPID" and FP7HEALTH-F3-2012-306029 “TRIGGER”), Polish Ministry of Science and Higher Education (project 2975/7.PR/13/2014/2) and internal project K/DSC/001820 (to MK). Faculty of Biochemistry, Biophysics and Biotechnology is a partner of the Leading National Research Center (KNOW) supported by the Ministry of Science and Higher Education.

\section{Supplementary Material}

The Supplementary Material for this article can be found online at: http://journal.frontiersin.org/article/10.3389/fmicb. 2015.00312/abstract
Bryan, P. N. (2002). Prodomains and protein folding catalysis. Chem. Rev. 102, 4805-4816. doi: 10.1021/cr010190b

Bryzek, D., Ksiazek, M., Bielecka, E., Karim, A. Y., Potempa, B., Staniec, D., et al. (2014). A pathogenic trace of Tannerella forsythia - shedding of soluble fully active tumor necrosis factor $\alpha$ from the macrophage surface by karilysin. Mol. Oral. Microbiol. 29, 294-306. doi: 10.1111/omi.12080

Burke, G. R., and Moran, N. A. (2011). Massive genomic decay in Serratia symbiotica, a recently evolved symbiont of aphids. Genome Biol. Evol. 3, 195-208. doi: 10.1093/gbe/evr002

Cerdà-Costa, N., Guevara, T., Karim, A. Y., Ksiazek, M., Nguyen, K. A., Arolas, J. L., et al. (2011). The structure of the catalytic domain of Tannerella forsythia karilysin reveals it is a bacterial xenologue of animal matrix metalloproteinases. Mol. Microbiol. 79, 119-132. doi: 10.1111/j.1365-2958.2010.07434.x

Colombo, A. P., Boches, S. K., Cotton, S. L., Goodson, J. M., Kent, R., Haffajee, A. D., et al. (2009). Comparisons of subgingival microbial profiles of refractory periodontitis, severe periodontitis, and periodontal health using the human oral microbe identification microarray. J. Periodontol. 80, 1421-1432. doi: 10.1902/jop.2009.090185 
Eick, S., Puklo, M., Adamowicz, K., Kantyka, T., Hiemstra, P., Stennicke, H., et al. (2014). Lack of cathelicidin processing in Papillon-Lefévre syndrome patients reveals essential role of LL-37 in periodontal homeostasis. Orphanet. J. Rare. Dis. 9:148. doi: 10.1186/s13023-014-0148-y

Guevara, T., Ksiazek, M., Skottrup, P. D., Cerdà-Costa, N., Trillo-Muyo, S., de Diego, I., et al. (2013). Structure of the catalytic domain of the Tannerella forsythia matrix metallopeptidase karilysin in complex with a tetrapeptidic inhibitor. Acta Crystallogr. Sect. F Struct. Biol. Cryst. Commun. 69, 472-476. doi: 10.1107/S1744309113007392

Guo, Y., Nguyen, K. A., and Potempa, J. (2010). Dichotomy of gingipains action as virulence factors: from cleaving substrates with the precision of a surgeon's knife to a meat chopper-like brutal degradation of proteins. Periodontol. 2000 54, 15-44. doi: 10.1111/j.1600-0757.2010.00377.x

Hamlet, S. M., Ganashan, N., Cullinan, M. P., Westerman, B., Palmer, J. E., and Seymour, G. J. (2008). A 5-year longitudinal study of Tannerella forsythia prtH genotype: association with loss of attachment. J. Periodontol. 79, 144-149. doi: 10.1902/jop.2008.070228

Imamura, T., Travis, J., and Potempa, J. (2003). The biphasic virulence activities of gingipains: activation and inactivation of host proteins. Curr. Protein Pept. Sci. 4, 443-450. doi: 10.2174/1389203033487027

Ishihara, K. (2010). Virulence factors of Treponema denticola. Periodontol. 2000 54, 117-135. doi: 10.1111/j.1600-0757.2009.00345.x

Jeftha, A., and Holmes, H. (2013). Periodontitis and cardiovascular disease. SADJ. 60, 62-63.

Jusko, M., Potempa, J., Karim, A. Y., Ksiazek, M., Riesbeck, K., Garred, P., et al. (2012). A metalloproteinase karilysin present in the majority of Tannerella forsythia isolates inhibits all pathways of the complement system. J. Immunol. 188, 2338-2349. doi: 10.4049/jimmunol.1101240

Karim, A. Y., Kulczycka, M., Kantyka, T., Dubin, G., Jabaiah, A., Daugherty, P. S., et al. (2010). A novel matrix metalloprotease-like enzyme (karilysin) of the periodontal pathogen Tannerella forsythia ATCC 43037. Biol. Chem. 391, 105-117. doi: 10.1515/bc.2010.009

Koziel, J., Karim, A. Y., Przybyszewska, K., Ksiazek, M., Rapala-Kozik, M., Nguyen, K. A., et al. (2010). Proteolytic inactivation of LL-37 by karilysin, a novel virulence mechanism of Tannerella forsythia. J. Innate. Immun. 2, 288-293. doi: 10.1159/000281881

Koziel, J., Mydel, P., and Potempa, J. (2014). The link between periodontal disease and rheumatoid arthritis: an updated review. Curr. Rheumatol. Rep. 16:408. doi: 10.1007/s11926-014-0408-9

Ksiazek, M., Karim, A. Y., Bryzek, D., Enghild, J. J., Thøgersen, I. B., Koziel, J., et al. (2015a). Mirolase, a novel subtilisin-like serine protease from the periodontopathogen Tannerella forsythia. Biol. Chem. 396, 261-275. doi: 10.1515/hsz2014-0256

Ksiazek, M., Mizgalska, D., Enghild, J. J., Scavenius, C., Thogersen, I. B., and Potempa, J. (2015b). Miropin, a novel bacterial serpin from the periodontopathogen Tannerella forsythia, inhibits a broad range of proteases by using different peptide bonds within the reactive center loop. J. Biol. Chem. 290, 658-670. doi: 10.1074/jbc.M114.601716

Lalla, E., and Papapanou, P. N. (2011). Diabetes mellitus and periodontitis: a tale of two common interrelated diseases. Nat. Rev. Endocrinol. 7, 738-748. doi: 10.1038/nrendo.2011.106

Lopez-Pelegrin, M., Ksiazek, M., Karim, A. Y., Guevara, T., Arolas, J. L., Potempa, J., et al. (2015). A novel mechanism of latency in matrix metalloproteinases. J. Biol. Chem. 290, 4728-4740. doi: 10.1074/jbc.M114.605956

Montagner, F., Jacinto, R. C., Signoretti, F. G., Sanches, P. F., and Gomes, B. P. (2012). Clustering behavior in microbial communities from acute endodontic infections. J. Endod. 38, 158-162. doi: 10.1016/j.joen.2011.09.029

Nakagawa, S., Niimura, Y., Miura, K., and Gojobori, T. (2010). Dynamic evolution of translation initiation mechanisms in prokaryotes. Proc. Natl. Acad. Sci. U.S.A. 107, 6382-6387. doi: 10.1073/pnas.1002036107

Nakajima, T., Tomi, N., Fukuyo, Y., Ishikura, H., Ohno, Y., Arvind, R., et al. (2006). Isolation and identification of a cytopathic activity in Tannerella forsythia. Biochem. Biophys. Res. Commun. 351, 133-139. doi: 10.1016/j.bbrc.2006.10.012

Narita, Y., Sato, K., Yukitake, H., Shoji, M., Nakane, D., Nagano, K., et al. (2014). Lack of a surface layer in Tannerella forsythia mutants deficient in the type IX secretion system. Microbiology 160, 2295-2303. doi: 10.1099/mic.0.080192-0

Nguyen, K. A., Travis, J., and Potempa, J. (2007). Does the importance of the Cterminal residues in the maturation of $\mathrm{RgpB}$ from Porphyromonas gingivalis reveal a novel mechanism for protein export in a subgroup of Gram-Negative bacteria? J. Bacteriol. 189, 833-843. doi: 10.1128/JB.01530-06

Nguyen, K. A., Zylicz, J., Szczesny, P., Sroka, A., Hunter, N., and Potempa, J. (2009). Verification of a topology model of PorT as an integral outermembrane protein in Porphyromonas gingivalis. Microbiology 155, 328-337. doi: 10.1099/mic.0.024323-0

Pei, J., and Grishin, N. V. (2009). Prediction of a caspase-like fold in Tannerella forsythia virulence factor PrtH. Cell Cycle. 8, 1453-1455. doi: $10.4161 / \mathrm{cc} .8 .9 .8243$

Pfaffl, M. W. (2001). A new mathematical model for relative quantification in real-time RT-PCR. Nucleic. Acids. Res. 29:e45. doi: 10.1093/nar/ 29.9.e45

Rawlings, N. D., Waller, M., Barrett, A. J., and Bateman, A. (2014). MEROPS: the database of proteolytic enzymes, their substrates and inhibitors. Nucleic. Acids. Res. 42, D503-D509. doi: 10.1093/nar/gkr987

Saito, T., Ishihara, K., Kato, T., and Okuda, K. (1997). Cloning, expression, and sequencing of a protease gene from Bacteroides forsythus ATCC 43037 in Escherichia coli. Infect. Immun. 65, 4888-3891.

Sato, K., Sakai, E., Veith, P. D., Shoji, M., Kikuchi, Y., Yukitake, H., et al. (2005). Identification of a new membrane-associated protein that influences transport/maturation of gingipains and adhesins of Porphyromonas gingivalis. J. Biol. Chem. 280, 8668-8677. doi: 10.1074/jbc.M413544200

Sato, K., Yukitake, H., Narita, Y., Shoji, M., Naito, M., and Nakayama, K. (2013). Identification of Porphyromonas gingivalis proteins secreted by the Por secretion system. FEMS Microbiol Lett. 338, 68-76. doi: 10.1111/ 1574-6968

Schägger, H., and von Jagow, G. (1987). Tricine-sodium dodecyl sulfatepolyacrylamide gel electrophoresis for the separation of proteins in the range from 1 to $100 \mathrm{kDa}$. Anal. Biochem. 166, 368-379. doi: 10.1016/0003-2697(87) 90587-2

Schatz, M. C., Delcher, A. L., and Salzberg, S. L. (2010). Assembly of large genomes using second-generation sequencing. Genome Res. 20, 1165-1173. doi: 10.1101/gr.101360.109

Schenkein, H. A. (2006). Host responses in maintaining periodontal health and determining periodontal disease. Periodontol. 2000 40, 77-93. doi: 10.1111/j.1600-0757.2005.00144.x

Sharma, A. (2010). Virulence mechanisms of Tannerella forsythia. Periodontol. 2000 54, 106-116. doi: 10.1111/j.1600-0757.2009.00332.x

Shoji, M., Sato, K., Yukitake, H., Kondo, Y., Narita, Y., Kadowaki, T., et al. (2011). Por secretion system-dependent secretion and glycosylation of Porphyromonas gingivalis hemin-binding protein 35. PLoS ONE 6:e21372. doi: 10.1371/journal.pone.0021372

Skorski, P., Leroy, P., Fayet, O., Dreyfus, M., and Hermann-Le Denmat, S. (2006) The highly efficient translation initiation region from the Escherichia coli rpsA gene lacks a shine-dalgarno element. J. Bacteriol. 188, 6277-6285. doi: 10.1128/JB.00591-06

Socransky, S. S., Haffajee, A. D., Cugini, M. A., Smith, C., and Kent, R. L. Jr. (1998). Microbial complexes in subgingival plaque. J. Clin. Periodontol. 25, 134-144. doi: 10.1111/j.1600-051X.1998.tb02419.x

Suzuki, J., Aoyama, N., Ogawa, M., Hirata, Y., Izumi, Y., Nagai, R., et al. (2010). Periodontitis and cardiovascular diseases. Expert Opin. Ther. Targets 14, 1023-1027. doi: 10.1517/14728222.2010.511616

Tanner, A. C., and Izard, J. (2006). Tannerella forsythia, a periodontal pathogen entering the genomic era. Periodontol. 2000 42, 88-113. doi: 10.1111/j.16000757.2006.00184.x

Tomek, M. B., Neumann, L., Nimeth, I., Koerdt, A., Andesner, P., Messner, P., et al. (2014). The S-layer proteins of Tannerella forsythia are secreted via a type IX secretion system that is decoupled from protein O-glycosylation. Mol. Oral Microbiol. 29, 307-320. doi: 10.1111/omi.12062

Tomi, N., Fukuyo, Y., Arakawa, S., and Nakajima, T. (2008). Pro-inflammatory cytokine production from normal human fibroblasts is induced by Tannerella forsythia detachingfactor. J. Periodontal Res. 43, 136-142. doi: 10.1111/j.16000765.2007.01003.x

Van Damme, P., Vandekerckhove, J., and Gevaert, K. (2008). Disentanglement of protease substrate repertoires. Biol. Chem. 389, 371-381. doi: 10.1515/BC. 2008.043

Veillard, F., Sztukowska, M., Mizgalska, D., Ksiazek, M., Houston, J., Potempa, B., et al. (2013). Inhibition of gingipains by their profragments as the 
mechanism protecting Porphyromonas gingivalis against premature activation of secreted proteases. Biochim. Biophys. Acta 1830, 4218-4228. doi: 10.1016/j.bbagen.2013.04.005

Veith, P. D., Nor Muhammad, N. A., Dashper, S. G., Likiæ, V. A., Gorasia, D. G., Chen, D., et al. (2013). Protein substrates of a novel secretion system are numerous in the Bacteroidetes phylum and have in common a cleavable C-terminal secretion signal, extensive post-translational modification, and cell-surface attachment. J. Proteome Res. 12, 4449-4461. doi: 10.1021/pr400487b

Veith, P. D., O'Brien-Simpson, N. M., Tan, Y., Djatmiko, D. C., Dashper, S. G., and Reynolds, E. C. (2009). Outer membrane proteome and antigens of Tannerella forsythia. J. Proteome Res. 8, 4279-4292. doi: 10.1021/pr900372c

Zhou, X. Y., Gao, J. L., Hunter, N., Potempa, J., and Nguyen, K. A. (2013). Sequence-independent processing site of the C-terminal domain (CTD) influences maturation of the RgpB protease from Porphyromonas gingivalis. Mol. Microbiol. 89, 903-917. doi: 10.1111/mmi.12319
Zuker, M. (2003). Mfold web server for nucleic acid folding and hybridization prediction. Nucleic Acids Res. 31, 3406-3415. doi: 10.1093/nar/ gkg595

Conflict of Interest Statement: The authors declare that the research was conducted in the absence of any commercial or financial relationships that could be construed as a potential conflict of interest.

Copyright (๐) 2015 Ksiazek, Mizgalska, Eick, Thøgersen, Enghild and Potempa. This is an open-access article distributed under the terms of the Creative Commons Attribution License (CC BY). The use, distribution or reproduction in other forums is permitted, provided the original author(s) or licensor are credited and that the original publication in this journal is cited, in accordance with accepted academic practice. No use, distribution or reproduction is permitted which does not comply with these terms. 